\title{
gp130 receptor ligands as potential therapeutic targets for obesity
}

\author{
Mark A. Febbraio
}

Cellular and Molecular Metabolism Laboratory, Division of Diabetes and Metabolism, Baker Heart Research Institute, Melbourne, Victoria, Australia.

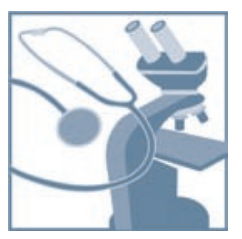

Obesity and its related cluster of pathophysiologic conditions including insulin resistance, glucose intolerance, dyslipidemia, and hypertension are recognized as growing threats to world health. It is now estimated that $10 \%$ of the world's population is overweight or obese. As a result, new therapeutic options for the treatment of obesity are clearly warranted. Recent research has focused on the role that gp130 receptor ligands may play as potential therapeutic targets in obesity. One cytokine in particular, ciliary neurotrophic factor (CNTF), acts both centrally and peripherally and mimics the biologic actions of the appetite control hormone leptin, but unlike leptin, CNTF appears to be effective in obesity and as such may have therapeutic potential. In addition, CNTF suppresses inflammatory signaling cascades associated with lipid accumulation in liver and skeletal muscle. This review examines the potential role of gp130 receptor ligands as part of a therapeutic strategy to treat obesity.

The prevalence of adult obesity has increased approximately $75 \%$ in the last quarter century (1). The prevalence of children who are overweight and obese is also increasing in both developed and developing countries (2). Among the disorders associated with obesity, insulin resistance, glucose intolerance, and dyslipidemia promote the development of pancreatic $\beta$ cell failure and overt type 2 diabetes. Current therapies to treat obesity center on lifestyle modifications, but for those individuals who do not respond to such treatment or cannot adhere to lifestyle intervention programs, bariatric surgery is often used. As this is neither a feasible nor a desirable treatment for a pandemic, drug therapy is a viable intervention for those in whom lifestyle modification has failed. Currently, there are 3 obesity drugs commonly prescribed. Xenical (orlistat) is a gastrointestinal lipase inhibitor known to cause moderate weight loss, but is associated with adverse gastrointestinal effects including colon cancer (3). Sibutramine, a monoamine reuptake inhibitor, results in greater weight loss compared with orlistat, but is associated with increased blood pressure and elevated heart rate. Rimonabant, the first of the endocannabinoid receptor antagonists, appears most promising, as it induces weight loss and improves waist circumference and HDL cholesterol concentration, but this drug is associated with the incidence of moodrelated disorders (4). Disappointingly, none have resulted in consistent and effective weight loss, and to date, all antiobesity drug trials have been limited by their high attrition rates and lack of long-term morbidity and mortality data (4). Current therapeutic strategies for diabetes and related diseases such as insulin resistance usually involve the administration of thiazolidinediones (TZDs) in combination with other therapies. These too have many disadvantages, including weight gain, particularly when TZDs and insulin are coadministered (5). Access to a drug capable of concomitantly decreasing body weight while enhancing insulin action

Nonstandard abbreviations used: ACC, acetyl-CoA carboxylase; AMPK, AMP kinase; CNTF, ciliary neurotrophic factor; CNTFR $\alpha$, CNTF receptor $\alpha$; gp130R, gp130 receptor; IL-6R, IL-6 receptor; LIF, leukemia inhibitory factor; LIFR $\beta$, LIF receptor $\beta$; LRb, long form of the leptin receptor; PGC- $1 \alpha$, PPAR $\gamma$ coactivator $1 \alpha$; POMC, proopiomelanocortin; SHP-2, Src homology-containing tyrosine phoshatase 2.

Conflict of interest: The author has declared that no conflict of interest exists. Citation for this article: J. Clin. Invest. 117:841-849 (2007). doi:10.1172/JCI30453. that does not induce adverse side effects such as those described above would have broad clinical impact.

\section{Leptin: the great panacea}

The existence of a molecule capable of signaling from fat to the CNS was hypothesized for several decades, and the discovery of leptin provided a critical scientific breakthrough in obesity-related disease research (6-9). The isolation of a molecule that could profoundly affect central feeding behavior led to the identification of canonical signaling pathways in the central regulation of energy homeostasis (10-12). A decade later, it is now well known that leptin primarily signals though the JAK/STAT pathway. Indeed, it was initially thought that leptin's effects were mediated exclusively via this signaling cascade, which ultimately causes alterations in the transcription of genes mediating behavioral and metabolic processes (13). However, many key metabolic effects of leptin signaling take place too rapidly to be ascribed solely to changes in processes in response to altered gene expression (14). Recent work from Minokoshi, Kahn, and colleagues has, for example, demonstrated that leptin acts on the brain by directly downregulating AMP kinase (AMPK) activity independent of changes to gene transcription (15). This is thought to be a direct, JAK/STAT-independent event. In recent years, work from several groups (16-18) has also demonstrated that leptin can increase fat oxidation and increase glucose uptake in skeletal muscle via activation of AMPK within skeletal muscle. The reason why leptin can reduce AMPK activity in the brain but activate it in the periphery is not completely clear, but may be related to differential actions of leptin on upstream kinases (14).

The discovery of leptin $(4,5)$ and the leptin receptor (19), the latter of which has both a long, full-length form (LRb) and a short, truncated form (20), led to the hope that researchers had at last identified a highly effective molecule and/or pathway that could be targeted in the treatment of obesity. However, it soon became apparent that obesity (in which high circulating concentrations of leptin develop) resulted in leptin resistance in the CNS, whereby endogenous leptin was no longer effective (21). Several mechanisms have been suggested to account for this resistance. The first appears to be related to reduced transport of leptin across the blood-brain barrier, since leptin maintains its effect when deliv- 


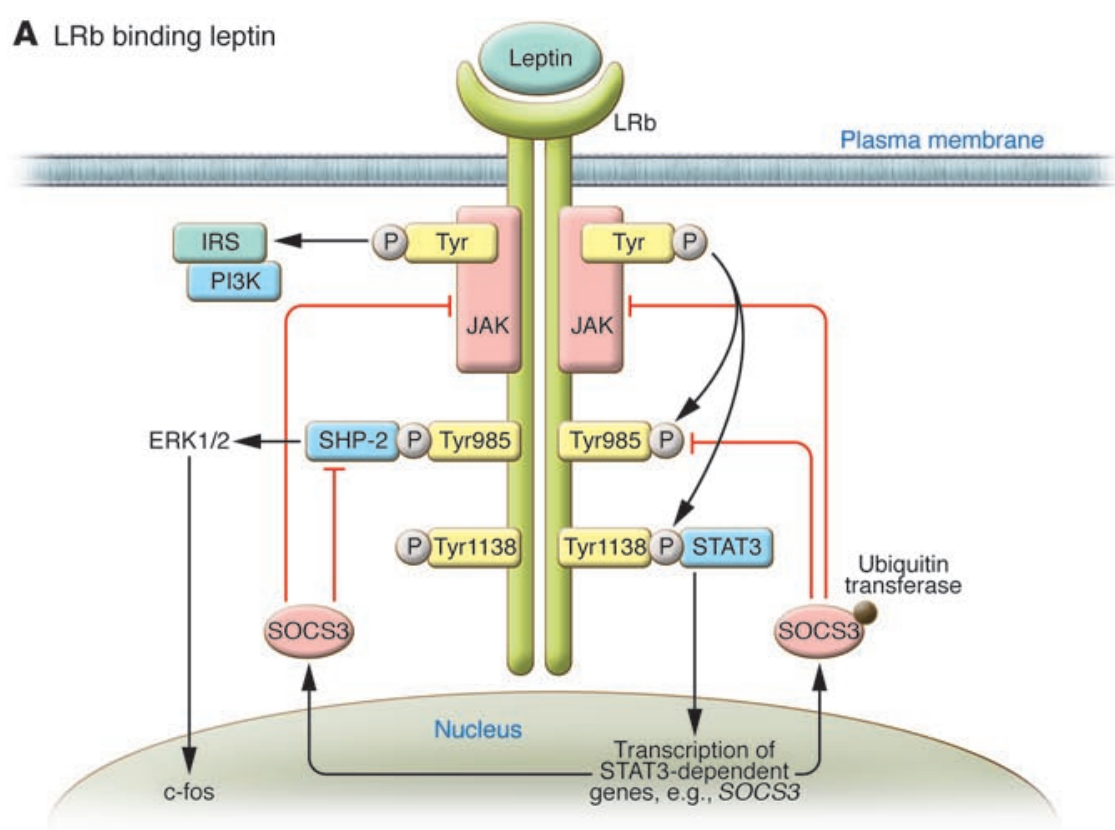

B IL-6R $\alpha / g p 130 R \beta$ homodimer binding IL-6 ligand

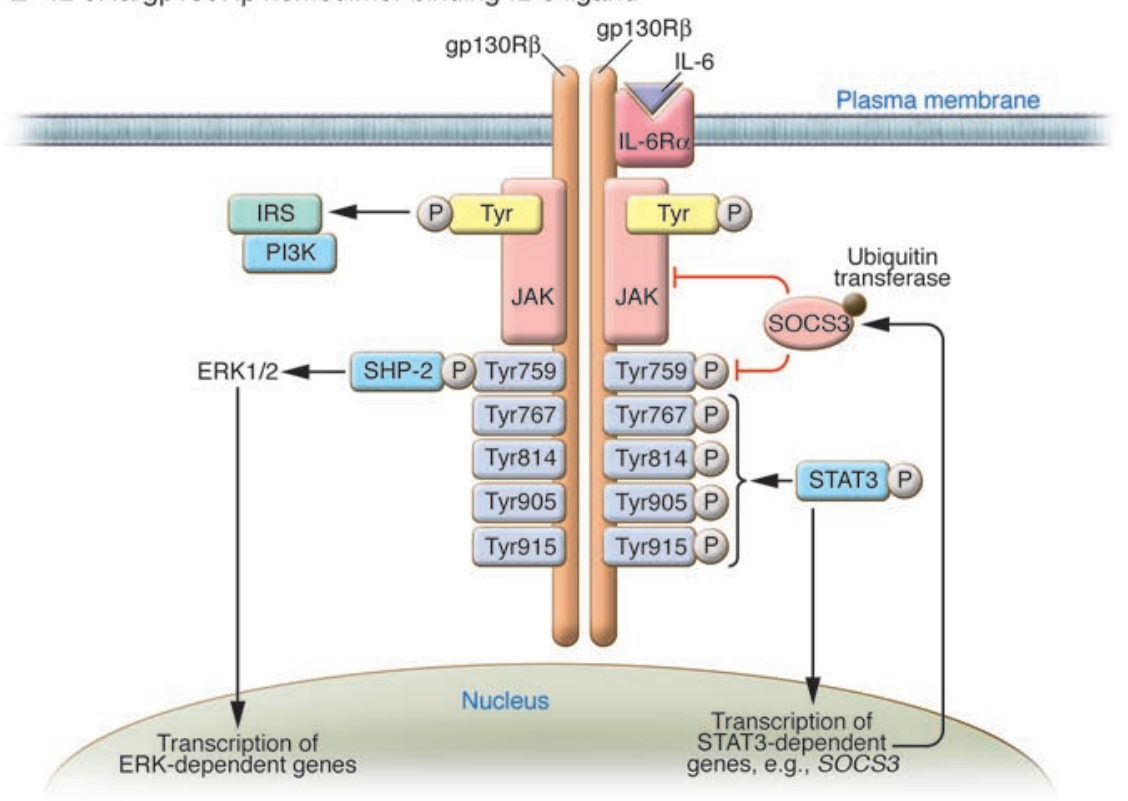

\section{Figure 1}

Signaling via LRb and gp130R: similarities and differences. (A) Leptin binds its homodimeric receptor LRb, which results in autophosphorylation and activation of JAK, subsequently activating the insulin receptor substrate/PI3K (IRS/PI3K) signalling pathway. JAK activation also results in the phosphorylation of $\mathrm{LRb}$ at Tyr985 and Tyr1138. The phosphorylation of Tyr1138 mediates the recruitment, phosphorylation, and activation of the transcription factor STAT3, resulting in the transcription of SOCS3 and other STAT3-dependent genes in the nucleus. SOCS3 inhibits leptin signaling via binding to SHP-2 bound to the LRb, recruiting ubiquitin transferases to the SOCS3 box domain at Tyr985; and binding JAK. (B) Signaling through gp $130 R \beta$ is similar to that through LRb. In the case of IL- 6 signaling, IL- 6 binds to the IL-6R $\alpha / g p 130 R \beta$ homodimer, which results in JAK/STAT, insulin receptor substrate/PI3K, and ERK signaling. Importantly, however, there are 4 tyrosine phosphorylation sites (at residues $767,814,905$, and 915 ) distal to the SHP-2 domain bound at Tyr759. As with the LRb, SOCS3 can inhibit JAK signaling on gp130R $\beta$. It is not clear why gp130R ligands may overcome SOCS3 inhibition, but it may be due to the 4 additional STAT3 binding sites, since truncation of gp130R to remove these sites does not allow ligand-mediated STAT3 phosphorylation in mice in vivo (18). ered centrally, but not peripherally, in diet-induced obese mice (22). Moreover, in obese humans, who have relative high circulating leptin levels, the uptake of leptin by the CNS (as determined by the cerebrospinal fluid/plasma leptin ratio) is compromised, demonstrating reduced efficiency of brain leptin delivery (23). The second mechanism is related to a novel cytokine-inducible molecule, suppressor of cytokine signaling (SOCS), which negatively regulates leptin signaling and leads to leptin resistance by binding Src homology-containing tyrosine phoshatase 2 (SHP-2) bound to phosphorylation sites on the active leptin receptor and inhibiting JAK tyrosine kinase activity via its N-terminal kinase motif (24-31) (Figure 1A). In mice haploinsufficient for SOCS3, leptin sensitivity is increased and high-fat diet-induced obesity is attenuated (27). Because elevated SOCS expression $(32,33)$ and leptin resistance
$(34,35)$ occur in both obese humans $(33,34)$ and rodents $(32,35)$, leptin's efficacy as an antiobesity drug appears limited to individuals with genetic mutations that result in leptin deficiency (36) or in patients undergoing sustained weight loss (37).

\section{Links to leptin signaling: gp130 cytokines}

The mRNA encoding IL- 6 was identified almost 25 years ago (38), and the gene was subsequently cloned and structurally characterized $(39,40)$. It is now known that a family of IL- 6 cytokines exists whose membership is based not on sequence homology, but on a shared 4-helical bundle structure (41) and a shared subunit in their respective receptor complexes: the transmembrane signal transduction protein gp130 (42). The IL-6 cytokine family - also known as long type I or gp130 cytokines - consists of ciliary neu- 

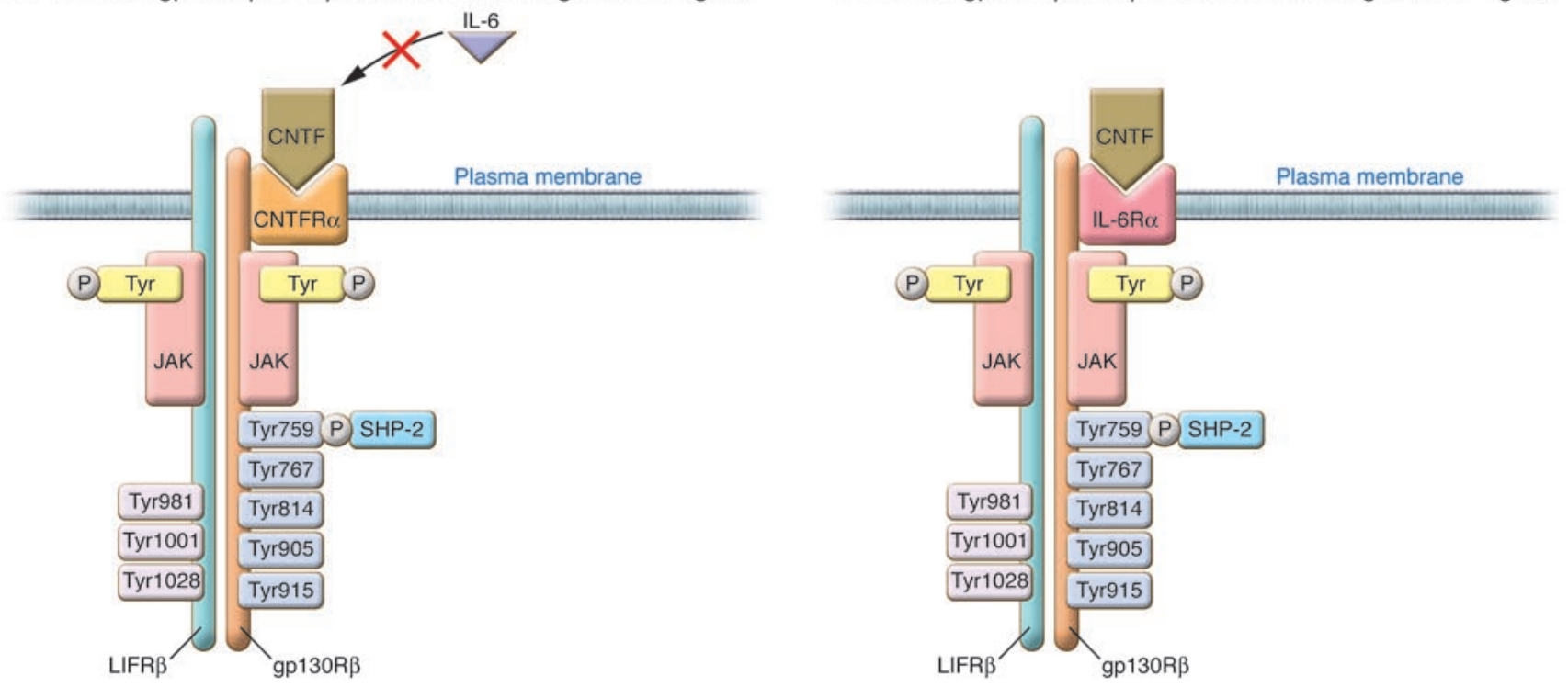

Figure 2

The complex ligand receptor signaling for the gp130R $\beta$ cytokine CNTF. In contrast to the mechanism of IL-6R $\alpha / g p 130 R \beta$ homodimer binding of IL-6 ligand illustrated in Figure 1B, CNTF can signal via a heterodimer containing CNTFR $\alpha$, gp 130R $\beta$, and LIFR $\beta$ (A) or via a heterodimer containing IL-6R $\alpha$, gp130R $\beta$, and LIFR $\beta$ (B). While CNTF can signal via IL-6R $\alpha$, it cannot do so via the IL-6R $\alpha / g p 130 R \beta$ homodimer shown in Figure $1 \mathrm{~B}$, as LIFR $\beta$ is an absolute requirement. In contrast, IL-6 does not signal through the CNTFR $\alpha / g p 130 R \beta / L I F R \beta$ heterodimer (51).

rotrophic factor (CNTF) as well as IL-6, IL-11, leukemia inhibitory factor (LIF), oncostatin M (OsM), cardiotrophin 1 (CT-1), and cardiotrophin-like cytokine (CLC) (43). Although there is some cross-talk among the gp130 cytokines (44), the complex signal transduction cascade is not common to all family members. IL- 6 and IL- 11 are the only members of the family that signal via induction of a gp130 homodimer after binding to IL- 6 receptor $\alpha$ (IL-6R $\alpha$ ) and IL-11R $\alpha$, respectively (Figure 1B). In contrast, CNTF, CT-1, and CLC first bind to their specific $\alpha$ receptors, which although the receptors are not involved in signal transduction per se - induces formation of a heterodimer of the signal-transducing gp130 receptor $\beta$ (gp130R $\beta$ ) and LIF receptor $\beta$ (LIFR $\beta$ ) to allow signal transduction (Figure $2 \mathrm{~A}$ ). LIF and OsM directly induce formation of gp130R $\beta /$ LIFR $\beta$ and gp130R $\beta / O s M$ receptor heterodimers, respectively (45). The gp130R $\beta$ in isolation cannot transduce signals without other specific $\alpha$ receptor subunits; therefore, although gp130 is ubiquitously expressed across all mammalian cell types, cell-specific responses to gp130 cytokines are dependent upon the relative expression of the $\alpha$ receptor within a cell type.

There are several reasons why a discussion of the intricacies of the complex ligand-receptor interactions of gp130 cytokines is pertinent to a discussion of antiobesogenic targets. LRb and gp130R $\beta$ share a large degree of sequence homology, and both activate the JAK/STAT and ERK signaling pathways (45). In addition, signaling through these receptors activates subsequent signal transduction pathways important in energy balance such as PI3K $(46,47)$ and $\operatorname{mTOR}(48,49)$. It must be noted, however, that $\mathrm{LRb}$ and gP130R $\beta$ cannot bind the other's specific ligand; the actions of CNTF are not compromised in the $d b / d b$ mouse (which lacks the functional LRb; ref. 18), and circulating leptin is normal in brain-specific gp130R $\beta$ knockout mice (50). Importantly, gp130R ligands may provide a way to circumvent leptin resis- tance, because while gp130R $\beta$ and LRb have a degree of sequence homology, gp130R $\beta$ has multiple tyrosine phosphorylation sites that may allow for adequate signaling in the obese phenotype, in which SOCS proteins are elevated. Finally, IL-6R can serve as an $\alpha$ receptor for CNTF (51) (Figure $2 \mathrm{~B}$ ). The importance of this is discussed below (see "Using gp130R ligands as therapy: where to now?"). Despite the fact that all gp130R cytokines signal through a common transmembrane receptor, most research pertaining to obesity-related disorders has centered upon IL-6 and CNTF.

\section{Biologic role of IL- 6 and its relationship to insulin resistance}

In the past decade it has become apparent that obesity is associated with a state of chronic inflammation (52) in tissue such as the liver, adipose, and smooth and skeletal muscle. Inflammation is characterized by the secretion of inflammatory cytokines such as resistin, TNF- $\alpha$, and IL- 6 from tissue-resident macrophages and/or adipocytes (53). Given this proinflammatory state, and the observation that systemic IL- 6 concentrations are elevated in obesity and patients with type 2 diabetes $(54,55)$, it is generally thought that elevations in the plasma and/or tissue concentrations of IL-6 lead to insulin resistance (56). Indeed, there are a number of studies both in vitro (57-60) and in rodents in vivo (61-63) that demonstrate that IL- 6 is capable of inducing insulin resistance. However, evidence is emerging that IL- 6 can in some circumstances activate pathways to promote increased energy turnover and insulin sensitivity, and the role of IL- 6 in the determination of insulin sensitivity is now a source of controversy $(64,65)$. While this notion challenges the dogma that IL- 6 is an inflammatory cytokine that has adverse metabolic effects, it is not inconceivable; as described above, gp130 cytokines signal through a receptor that has many similarities to leptin signaling, and leptin is known to activate 
signal transduction pathways that promote increased energy expenditure and insulin sensitivity (21). In exercising rats, hypothalamic leptin and insulin sensitivity are increased in an IL-6dependent manner (66). In 3 recent studies, IL- 6 has been shown (like leptin) to activate AMPK in both skeletal muscle and adipose tissue (67-69). Consistent with activation of AMPK, IL-6 has also been shown to increase fat oxidation in vitro (60-70), ex vivo (71), and in humans in vivo $(70,72)$, while inactivation of AMPK using siRNA (68) or a dominant-negative adenovirus (69) abolishes the effect of IL- 6 on fat oxidation. Signaling through gp $130 \mathrm{R} \beta$ results in activation of PI3K (47), and IL- 6 can activate PI3K and its downstream target Akt $(68,73-75)$, but it must be noted that this effect has not been observed in all studies (69). Given that IL-6 activates the immune system and is proinflammatory, particularly in the liver $(61,62)$, it is clear that recombinant human IL-6 treatment would not be a wise therapeutic strategy to treat obesity. However, given the recent evidence that IL- 6 and leptin have common signal transduction pathways downstream of signaling through gp130R $\beta$ and LRb, respectively, it is possible that other endogenous or synthetic gp130R agonists may be viable antiobesity therapeutics.

\section{Can CNTF circumvent leptin resistance and result in weight control?}

CNTF was first identified over 25 years ago for its ability to support the in vitro survival of chick ciliary ganglion neurons during maturation (76). It was subsequently purified from sciatic nerves by 2 groups a decade later $(77,78)$. CNTF is described as a $22-\mathrm{kDa}$, 200 -aa-long member of the IL- 6 family of cytokines, sharing the 4-helical bundle structure (79). As a neurotrophic factor it promotes differentiation of sympathetic neurons and glial progenitor cells into astrocytes; supports the survival of sensory, motor, hippocampal, and cerebral neurons; and promotes the maturation of oligodendrocytes (79). However, unlike IL-6, CNTF lacks a signal sequence peptide and is therefore not secreted by neuronal cells $(80,81)$, which has therapeutic implications (see "Using gp130R ligands as therapy: where to now?”). CNTF receptor $\alpha$ (CNTFR $\alpha$ ) is most highly expressed within neural tissue, but its expression is ubiquitous, being detected in the adrenal glands, liver, kidney, testis, and skin (79). It is important to note that relative to its expression in other peripheral tissues, CNTFR $\alpha$ is also highly expressed in skeletal muscle (80), although its mRNA expression in both fastand slow-twitch muscle is approximately 6 -fold lower than that of $I L-6 R \alpha$ (18). The expression of CNTFR $\alpha$ in skeletal muscle appears to be functionally important, as CNTF has recently been shown to be capable of inducing the dedifferentiation of adult human myoblasts into multipotent progenitor cells (81) and preventing loss of muscle strength associated with aging (82). Prior to the discovery that gp130R and LRb shared sequence homology and a common JAK/STAT signaling cascade, the antiobesogenic properties of CNTF treatment were uncovered in a study of patients with amyotrophic lateral sclerosis (ALS). As a neurotrophic factor, CNTF was used in a clinical trial in an attempt to decrease disease progression (83). While this proved unsuccessful, the patients, who were not classified as obese at the onset of the trial, experienced marked involuntary weight loss. This finding provided the impetus for a number of studies examining the efficacy of CNTF as an antiobesity drug and ultimately the development of $\mathrm{CNTF}_{\mathrm{Ax} 15}$ (also known as Axokine), the human recombinant form of CNTF. Compared with the parent molecule, $\mathrm{CNTF}_{\mathrm{Ax} 15}$ has a cystine replaced by an alanine at position 17 , a glutamine replaced by an arginine at position 63, and a truncation of the last 15 amino acids at the $\mathrm{N}$ terminus. These modifications increased its stability, potency, and solubility (79). Human clinical trials with $\mathrm{CNTF}_{\mathrm{Ax} 15}$ are discussed below (see "CNTF/CNTFR $\alpha$ mutations in humans and efficacy of $\mathrm{CNTF}_{\mathrm{Ax} 15}$ in clinical trials”).

\section{Central effects of CNTF}

The first report that CNTF might be a strategic target for the treatment of obesity associated with leptin resistance was published a decade ago. Gloaguen and colleagues (84) reported that CNTFR $\alpha$ and LRb were colocalized in the hypothalamic region of the brain involved in the regulation of energy balance. In addition, systemic administration of either CNTF or leptin activated and suppressed the same genes in cell bodies of the arcuate nucleus, suggesting that both cytokines were capable of anorexogenic neuronal signaling. Moreover, administration of CNTF in leptin-resistant models of obesity, namely $o b / o b, d b / d b$, and high-fat-fed mice, was found to result in hypophagia, reduced body weight, and attenuated hyperinsulinemia. This initial study was confirmed by Lambert et al. (85), who showed that $\mathrm{CNTF}_{\mathrm{Ax} 15}$ corrected the obese phenotype in leptin-resistant rodent models without side effects such as fever and other metabolic abnormalities often associated with cytokine treatment (86). Whether $\mathrm{CNTF}$ or $\mathrm{CNTF}_{\mathrm{Ax} 15}$ treatment results in a proinflammatory response is equivocal, as subsequent studies have reported the presence $(87,88)$ and absence $(18)$ of fever as well as upregulation of proinflammatory gene expression following administration.

It is important to note that in the study by Lambert et al. (85), animals continued to maintain a reduced body weight several days following the cessation of treatment. This suggested that the mode of action of CNTF was not solely though the activation of the tripartite CNTFR $\alpha / g p 130 R \beta / L I F R \beta$ complex. Until recently, the mechanism underlying CNTF- or $\mathrm{CNTF}_{\mathrm{Ax} 15}$-related weight gain prevention observed after the cessation of treatment remained elusive. However, Flier and colleagues recently demonstrated that centrally administered CNTF leads to cell proliferation in the hypothalamus of mice (89), the region of the brain that contains neurons such as proopiomelanocortin (POMC) and neuropeptide Y (NPY), which are known to play a role in energy balance regulation (90). The hypothalamic neurogenesis was prevented by coadministration of the mitotic blocker cytosine $\beta$-D-aribinofuranoside, which was accompanied by rebound weight gain (89). This concept of neural growth provided a new dimension to strategies targeting weight loss because CNTF and other neurogenic compounds would allow for the growth of new leptin-responsive neurons in the hypothalamus, making the brain more sensitive to compounds that modulate POMC and NPY even after cessation of treatment. It must be noted, however, that these results have been challenged on the basis that the studies reported (89) did not categorically prove that the treatment resulted in full-fledged neurons, but rather supported the survival of immature but preexisting brain cells (91). Regardless of whether CNTF results in bona fide neurogenesis, it is clear that an important feature of CNTF or its analogs is the capacity to remain effective after termination of therapy, which may provide scope to trial drugs that could be used in a cyclic manner in the treatment of obesity. However, new cell growth in the CNS may lead to adverse side effects that could limit the efficacy of such treatment. Strategies to limit this potentially dangerous outcome are under development (see "Using gp130R ligands as therapy: where to now?"). 
The exact site of CNTF action in the arcuate has only recently been uncovered. Janoschek, Brüning, and colleagues (50) engineered mice with selective ablation of the gp130R in POMCexpressing neurons (gP130 ${ }^{\triangle \mathrm{POMC}}$ mice). When fed regular chow or a high-fat diet, the gp $130^{\triangle \mathrm{POMC}}$ mice displayed a normal phenotype, suggesting that endogenous CNTF plays no role in energy balance. However, in contrast with the expected response as seen in littermate control mice, the effect of centrally administered CNTF was abolished in gP130 ${ }^{\triangle \mathrm{POMC}}$ mice, identifying a neuronal circuit by which CNTF signals in the hypothalamus.

Some of leptin's central actions are mediated via inhibition of AMPK $(15,92)$. The central action of CNTF also involves the downregulation of AMPK, since both i.p. and icv injection of $\mathrm{CNTF}_{\mathrm{Ax} 15}$ decrease AMPK $\alpha 2$ activity (93). Moreover, icv administration of $\mathrm{CNTF}_{\mathrm{Ax} 15}$ into the lateral ventricle increases phosphorylation of STAT3, but reduces AMPK, and acetyl-CoA carboxylase (ACC) phosphorylation in the arcuate, while markedly reducing food intake and body weight in animals fed regular chow and/or a high-fat diet. Consistent with previous findings, the efficacy of leptin was abolished in animals fed the high-fat diet (93). In summary, CNTF and its analogs appear to be effective mediators of hypothalamic control of energy balance via multiple pathways. They appear to specifically activate POMC neural circuits in the arcuate and to decrease activation of AMPK.

\section{Peripheral effects of CNTF}

In one of the early studies showing that $\mathrm{CNTF}_{\mathrm{Ax} 15}$ prevented weight gain, the authors observed a greater weight loss in treated compared with pair-fed control animals (85). This observation suggested that CNTF or its analogs could increase energy expenditure independent of effects on energy intake. This study could not determine whether the increase in energy expenditure as a result of $\mathrm{CNTF}_{\mathrm{Ax} 15}$ administration was centrally mediated, but it raised the possibility that CNTF could exert some actions in peripheral tissues via a mechanism independent of central mediation. Two subsequent in vitro studies supported the concept that CNTF may act on peripheral target tissues in a manner independent of central mediation. CNTF was shown to increase the mRNA expression and/or phosphorylate several proteins in brown adipocytes (94) as well as in 3T3-L1 preadipocytes and mature adipocytes (95). In the latter study, the authors noted that this effect occurred in spite of the fact that adipocytes do not express CNTFR $\alpha$, suggesting that the IL-6R $\alpha$ may serve as an $\alpha$ receptor for CNTF in adipocytes (51). CNTF also activated STAT3 in the adipose tissue and skeletal muscle of mice in vivo, which suggested that CNTF could exert peripheral, central mediation-independent actions, but the study could not rule out the possibility that when administered i.p., CNTF was acting via a neural circuit. Although leptin was shown to directly activate AMPK in the periphery, central administration of leptin can also result in activation of AMPK in skeletal muscle (16). To test whether this could also be the case with CNTF, mice were treated with CNTF either i.p. or icv and skeletal muscle was excised. CNTF increased STAT3 phosphorylation and AMPK activity in skeletal muscle when delivered i.p., but not when delivered icv (18). This was the first report providing evidence that CNTF could act in a manner independent of central mediation. Consistent with the work conducted by this group on another gp130 cytokine, IL-6 (69), CNTF was able to increase fat oxidation in skeletal muscle via an AMPK-dependent mechanism, since the infection of muscle cells with an AMPK dominant-negative adenovirus abolished the
CNTF-mediated increase in fat oxidation. Mice treated with CNTF for 7 days lost more weight compared with sham-treated, pair-fed animals fed a high-fat diet, confirming previous in vivo observations (85). This weight loss was associated with improved insulin signal transduction and insulin action in skeletal muscle. The effects were also seen in $d b / d b$ mice. Importantly, these authors showed that while both leptin and CNTF caused activation of STAT3 and AMPK, phosphorylation of ACC, and enhanced fat oxidation in the skeletal muscle of mice ingesting a regular chow diet, only CNTF maintained these effects in mice fed a high-fat diet or in $d b / d b$ mice (18).

This recent study has heightened interest in the possibility of using CNTF or its analogs as a treatment for obesity (96) and has also uncovered a possible mechanism by which gp130R cytokines might overcome leptin resistance. As seen in Figures 1 and 2 , while the LRb and CNTFR $\alpha / g p 130 R \beta$ complexes share structural homology in their cytoplasmic domains, there are nonetheless important distinctions. Similar to the way in which SOCS proteins bind SHP-2 bound to LRb and attenuate leptin signaling, SOCS proteins can inhibit JAK/STAT signaling on the gp130R $\beta$ receptor by binding to the JAK site. However, it appears that CNTF can overcome SOCS inhibition of receptor signaling. While the capacity for CNTF to override SOCS inhibition of signal transduction has not been fully elucidated, it is hypothesized that this is due to the gp130R possessing 4 STAT-binding sites (at Tyr767, Tyr814, Tyr905, and Tyr915; Figure 2). This hypothesis is based on the following observation: when mice with a truncation of the C-terminal domain that eliminates these tyrosine residues of the gp130R (gp130 ${ }^{\triangle \mathrm{STAT}}$ mice) are treated with CNTF, STAT3 phosphorylation is abolished as well as AMPK activation, ACC phosphorylation, and ultimately fat oxidation (18). These results suggest that the STAT3-binding sites downstream of the SHP-2/ Tyr759 binding site on gp130R $\beta$ are functionally important for transducing signaling after ligand binding (Figure 2). Mutations of these 4 STAT bindings sites of gp130R $\beta$ are currently being synthesized. This work may determine the functional significance of these sites in the context of the capacity for gp130R signaling to overcome SOCS inhibition.

In mice, CNTF treatment also markedly attenuates high-fat dietinduced accumulation of lipid in skeletal muscle (18). Specifically, the intramuscular accumulation of triacylglycerol, diacylglycerol, and ceramide is restored to the level in control mice fed regular chow. Accumulation of lipid metabolites in peripheral tissues correlates with insulin resistance (97). In addition, the production of fatty acid metabolites within insulin-responsive tissues activates a host of serine kinases including PKC- $\theta$, Iк kinase, and JNK, which have been shown to impair insulin signal transduction and insulin action in vivo (98-100). CNTF may attenuate such lipotoxicity and resultant inflammatory serine kinase cascades. When rats were treated with $\mathrm{CNTF}_{\mathrm{Ax} 15}$ during a 2-hour lipid infusion, both skeletal muscle and liver insulin action were restored during a subsequent hyperinsulinemic euglycemic clamp (101). Moreover, CNTF treatment prevented acute, lipid-induced activation of JNK in skeletal muscle and both JNK and NF- $\mathrm{KB}$ in liver. This effect was associated with reduced fat deposition in these tissues, confirming earlier findings that CNTF can attenuate hepatic steatosis (102). When $d b / d b$ mice were treated with $\mathrm{CNTF}_{\mathrm{Ax} 15}$ for 10 days, liver steatosis was found to be reduced, which coincided with improvements in liver function and insulin signaling (102); the authors also observed increased basal metabolic rate of $d b / d b$ mice 


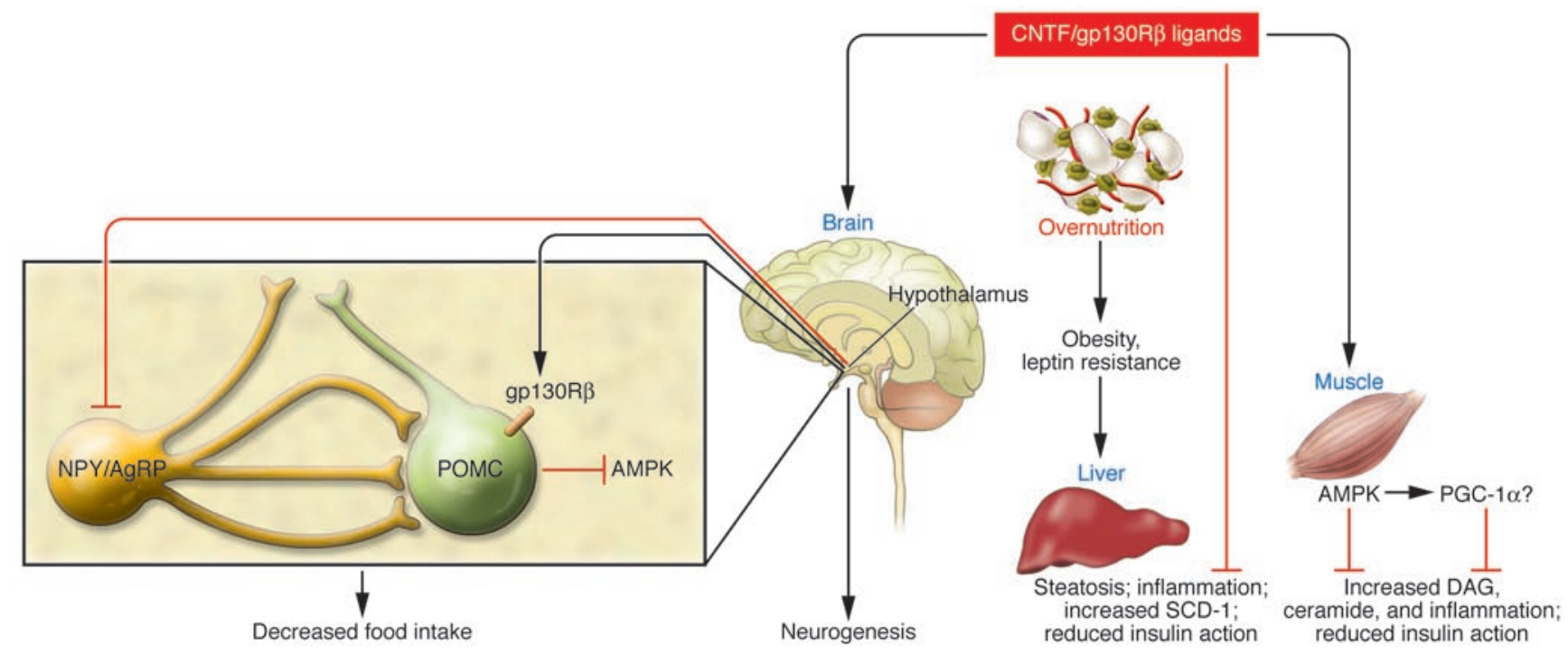

Figure 3

Pathways by which CNTF acts as an antiobesogenic and insulin-sensitizing agent. CNTF or other gp130R ligands can act in the CNS by signaling through the gp130R in POMC neurons in the hypothalamus to decrease AMPK and also by promoting neurogenesis in the arcuate nuclei. In the brain, CNTF/gp130R $\beta$ ligands can also reduce neuropeptide $Y(N P Y)$ gene expression. These actions result in a decrease in food intake. In the periphery, gp130R ligands can increase lipid oxidation, thereby preventing steatosis in the liver and lipid accumulation in the muscle. The enhanced fat oxidation in these tissues and resultant decrease in accumulation of deleterious lipid species (diacylglyceride [DAG] and ceramide) prevent the activation of inflammatory serine threonine kinase cascades (JNK and lא kinase) in the liver and muscle and the transcription of Stearoyl-CoA desaturase (SCD-1) in liver to ameliorate lipid-induced decreases in insulin signal transduction. Although speculative, in skeletal muscle, these actions appear to be mediated by activation of AMPK, which may also lead to activation of PGC-1 $\alpha$. AgRP, agouti-related protein.

treated with $\mathrm{CNTF}_{\mathrm{Ax} 15}$. This effect was confirmed by a subsequent study from this group (103) that also found increases in uncoupling protein 1 (UCP1) mRNA levels in brown adipose tissue. This latter observation is consistent with the finding that CNTF can increase the mRNA expression of the Ppargc1a gene and prevent the highfat diet-induced decline in its encoded protein, PPAR $\gamma$ coactivator $1 \alpha$ (PGC-1 $\alpha$ ), in skeletal muscle (18). A recent study has shown that AICAR, a pharmacologic activator of AMPK, increased PGC-1 $\alpha$ promoter levels in C2C12 muscle cells (104), and a number of studies have recently implicated defective mitochondria, specifically reduced PGC- $1 \alpha$-responsive gene expression and/or reduced mitochondrial ATP turnover, in the etiology of insulin resistance and type 2 diabetes $(105,106)$. Although speculative, one antiobesogenic action of gp130R cytokines may occur via an AMPK-mediated increase in PGC-1 $\alpha$ levels and subsequent increase in mitochondrial biogenesis (Figure 3); this hypothesis is currently being tested. Thus, in addition to the well-described central actions of CNTF and its analogs, these cytokines are capable of enhancing insulin action and promoting lipid oxidation in peripheral tissues (by apparent direct action in those tissues).

\section{CNTF/CNTFR $\alpha$ mutations in humans and efficacy of $\mathrm{CNTF}_{\mathrm{Ax} 15}$ in clinical trials}

A mutation in the first intron of the human CNTF gene was first identified over a decade ago (107). This mutation resulted in a novel splice acceptor site and mRNA encoding a novel protein. In addition, 3 single nucleotide polymorphisms (SNPs) in the gene encoding CNTFR $\alpha$ have been identified (108). Given the association of CNTF with energy homeostasis, studies have examined whether any of these mutations are associated with body mass homeostasis in humans. While some found no association between genetic markers segregating with CNTF and body weight regulation (109), others observed that in a cohort of elderly male Caucasians, carriers of the gene mutation are on average $10 \mathrm{~kg}$ heavier than those lacking the mutation (108). In addition, of the 3 identified SNPs in the gene encoding CNTFR $\alpha$, the C174T polymorphism in exon 9 is associated with body weight regulation in both men and women (110). In a phase II clinical trial of the effects of $\mathrm{CNTF}_{\mathrm{Ax} 15}$ administration, patients recruited to the study were on average approximately $114 \mathrm{~kg}$ with an average BMI of approximately 41. Over a period of 84 days, patients treated with a placebo maintained their weight, while patients treated with $\mathrm{CNTF}_{\mathrm{Ax} 15}$ at doses of 1 or $2 \mu \mathrm{g} / \mathrm{kg}$ body weight lost 3-4 kg. While these results were promising, the efficacy of $\mathrm{CNTF}_{\mathrm{Ax} 15}$ as a treatment appears limited, since patients treated with high doses of the drug reported nausea or developed anti-CNTF $\mathrm{Ax}_{15}$ antibodies. This effect may have been responsible for an increase in weight gain in $\mathrm{CNTF}_{\mathrm{Ax} 15^{-}}$ treated patients in a long-term follow-up study (111).

\section{Using gp130R ligands as therapy: where to now?}

Given the lack of success with current gp130R ligands in clinical trials, why should we pursue this class of proteins as possible antiobesity therapies? As discussed in this review, inflammation in the CNS $(86,87)$ and anti-CNTF antibody formation (112) are major hurdles that require attention. Nonetheless, recent results showing potent peripheral effects of gp130R $\beta$ ligands in increasing lipid oxidation, activating AMPK, preventing lipid-induced inflammation, and upregulating genes associated with oxidative phosphorylation $(18,68,69,101)$ have led to the suggestion that alternative treatment strategies using these ligands are possible (96). Impor- 
tantly, unlike its effects in the brain, CNTF has no adverse effects on markers of inflammation in peripheral tissue (18), and by virtue of reducing intracellular lipid accumulation in these tissues, CNTF can reduce the activation of inflammatory signaling pathways such as JNK and NF-кB (100). Hence, a drug capable of activating gp130R $\beta$ in the periphery alone could be a viable therapeutic strategy. As described above, IL-6R $\alpha$ can serve as an $\alpha$ receptor for CNTF (51). However, each ligand has a greater degree of binding affinity for their specific $\alpha$ receptor, and it must be emphasized that CNTFR $\alpha$ has a relatively low level of expression in skeletal muscle (18) and is undetectable in adipose tissue (95). With this in mind, a drug that could have the general properties of CNTF but specifically activate IL-6R $\alpha$ may be useful. Hence, the efficacy of so-called "designer gp130R ligands" is currently being studied. The design of these ligands is based on the observation that receptor recognition sites of gp130 cytokines are organized as exchangeable modules and various chimeras in which the site III loop of IL-6 has been substituted for the site III loop of CNTF (112). The site III loop is situated on the C-terminal end of the protein and is the region that binds the receptor (112). Thus, it is hypothesized that this designer gp130 chimera possesses CNTF-like action, but has a greater binding affinity for IL-6R $\alpha$, which, as discussed above, is much more highly expressed in peripheral tissues (18). Theoretically, therefore, the dose required to signal through the IL-6R $\alpha$ / gp130R $\beta$ homodimer would be markedly lower than that of CNTF and would be less likely to result in antibody formation. In addition, since the peripheral metabolic actions of gp130R ligands do not require CNS signaling, an attractive approach would be to fuse the ligand to a larger protein, thereby preventing it from crossing the blood-brain barrier. One such strategy being examined is to fuse the chimera to the Fc portion of IgG, a strategy used for many clinical therapeutics, including TNF inhibitors, for the treatment of rheumatoid arthritis (113). Whether designer gp130R ligands prove useful as antiobesity drugs remains to be seen, but given recent results showing potent peripheral effects of gp130R ligands, these strategies warrant further investigation.

\section{Acknowledgments}

I would like to acknowledge all past and present members of the Cellular and Molecular Metabolism Laboratory who have contributed to this work and all of my collaborators, especially Matthew Watt, Gregory Steinberg, Andrew Carey, Stefan Rose-John, Bruce Kemp, and Bente Klarlund Pedersen. The support from the Australian Health and Medical Research Council, the Australia Research Council, and the Diabetes Australia Research Trust is gratefully acknowledged.

Address correspondence to: Mark A. Febbraio, Baker Heart Research Institute, PO Box 6492, St. Kilda Road Central, Melbourne, Victoria 8008, Australia. Phone: 61-3-9925-7305; Fax: 613-9925-7083; E-mail: mark.febbraio@baker.edu.au.
1. Flegal, K.M., Carroll, M.D., Ogden, C.L., and Johnson, C.L. 2002. Prevalence and trends in obesity among US adults, 1999-2000. JAMA. 288:1723-1727.

2. Mascie-Taylor, C.G., and Karim, E. 2003. The burden of chronic disease. Science. 302:1921-1922.

3. Garcia, S.B., et al. 2006. The anti-obesity agent Orlistat is associated to increase in colonic preneoplastic markers in rats treated with a chemical carcinogen. Cancer Lett. 240:221-224.

4. Padwal, R.S., and Majumdar, S.R. 2007. Drug treatment for obesity: orlistat, sibutramine, and rimonbant. Lancet. 369:71-77.

5. Giorgino, F., Laviola, L., and Leonardini, A. 2005. Pathophysiology of type 2 diabetes: rationale for different oral antidiabetic treatment strategies. Diabetes Res. Clin. Pract. 68(Suppl. 1):S22-S29.

6. Zhang, Y., et al. 1994. Positional cloning of the mouse obese gene and its human homologue. Nature. 372:425-432.

7. Halaas, J.L., et al. 1995. Weight-reducing effects of the plasma protein encoded by the obese gene. Science. 269:543-546.

8. Kennedy, G.C. 1953. The role of depot fat in the hypothalamic control of food intake in the rat. Proc. R. Soc. Lond. B Biol. Sci. 140:578-592.

9. Coleman, D.L. 1973. Effects of parabiosis of obese with diabetes and normal mice. Diabetologia. 9:294-298

10. Elmquist, J.K., et al. 1998. Leptin activates distinct projections from the dorsomedial and ventromedial hypothalamic nuclei. Proc. Natl. Acad. Sci. U. S. A. 95:741-746.

11. Friedman, J.M., and Halaas, J.L. 1998. Leptin and the regulation of body weight in mammals. Nature. 395:763-770.

12. Schwartz, M.W., Erickson, J.C., Baskin, D.G., and Palmiter, R.D. 1998. Effect of fasting and leptin deficiency on hypothalamic neuropeptide $Y$ gene transcription in vivo revealed by expression of a lacZ reporter gene. Endocrinology. 139:2629-2635.

13. Bjørbæk, C., and Kahn, B.B. 2004. Leptin signaling in the central nervous system and the periphery. Recent Prog. Horm. Res. 59:305-331.

14. Kahn, B.B., Alquier, T., Carling, D., and Hardie,
D.G. 2005. AMP-activated protein kinase: ancient energy gauge provides clues to modern understanding of metabolism. Cell Metab. 1:15-26.

15. Minokoshi, Y., et al. 2004. AMP-kinase regulates food intake by responding to hormonal and nutrient signals in the hypothalamus. Nature. 428:569-574.

16. Minokoshi, Y., et al. 2002. Leptin stimulates fattyacid oxidation by activating AMP-activated protein kinase. Nature. 415:339-343.

17. Steinberg, G.R., Rush, J.W., and Dyck, D.J. 2003. AMPK expression and phosphorylation are increased in rodent muscle after chronic leptin treatment. Am. J. Physiol. Endocrinol. Metab. 284:E648-E654.

18. Watt, M.J., et al. 2006. CNTF reverses obesityinduced insulin resistance by activating skeletal muscle AMPK. Nat. Med. 12:541-548.

19. Tartaglia, L.A., et al. 1995. Identification and expression cloning of a leptin receptor, OB-R. Cell. 83:1263-1271.

20. Baumann, H., et al. 1996. The full-length leptin receptor has signaling capabilities of interleukin 6 type cytokine receptors. Proc. Natl. Acad. Sci. U. S. A. 93:8374-8378.

21. Flier, J.S. 2004. Obesity wars: molecular progress confronts an expanding epidemic. Cell. 116:337-350.

22. Van Heek, M., et al. 1997. Diet-induced obese mice develop peripheral, but not central, resistance to leptin. J. Clin. Invest. 99:385-390.

23. Schwartz, M.W., et al. 1996. Cerebrospinal fluid leptin levels: relationship to plasma levels and to adiposity in humans. Nat. Med. 2:589-593.

24. Naka, T., et al. 1997. Structure and function of a new STAT-induced STAT inhibitor. Nature. 387:924-929.

25. Yoshimura, A., et al. 1995. A novel cytokine-inducible gene CIS encodes an SH2-containing protein that binds to tyrosine-phosphorylated interleukin 3 and erythropoietin receptors. EMBOJ. 14:2816-2826.

26. Starr, R., et al. 1997. A family of cytokine-inducible inhibitors of signalling. Nature. 387:917-921.

27. Howard, J.K., et al. 2004. Enhanced leptin sensitivity and attenuation of diet-induced obesity in mice with haploinsufficiency of Socs3. Nat. Med. 10:734-738.
28. Mori, H., et al. 2004. Socs3 deficiency in the brain elevates leptin sensitivity and confers resistance to diet-induced obesity. Nat. Med. 10:739-743.

29. Yasukawa, H., Sasaki, A., and Yoshimura, A. 2000. Negative regulation of cytokine signaling pathways. Annu. Rev. Immunol. 18:143-164.

30. Bjørbæk, C., et al. 2000. Divergent roles of SHP-2 in ERK activation by leptin receptors. J. Biol. Chem. 276:4747-4755.

31. Eyckerman, S., et al. 2000. Identification of the Y985 and Y1077 motifs as SOCS3 recruitment sites in the murine leptin receptor. FEBS Lett. 486:33-37.

32. Ueki, K., Kondo, T., Tseng, Y.H., and Kahn, C.R. 2004. Central role of suppressors of cytokine signaling proteins in hepatic steatosis, insulin resistance, and the metabolic syndrome in the mouse. Proc. Natl. Acad. Sci. U. S. A. 101:10422-10427.

33. Steinberg, G.R., Parolin, M.L., Heigenhauser, G.J., and Dyck, D.J. 2002. Leptin increases FA oxidation in lean but not obese human skeletal muscle: evidence of peripheral leptin resistance. Am. J. Physiol. Endocrinol. Metab. 283:E187-E192.

34. Considine, R.V., et al. 1996. Serum immunoreactive-leptin concentrations in normal-weight and obese humans. N. Engl. J. Med. 334:292-295.

35. Frederich, R.C., et al. 1995. Leptin and the regulation of body weight in mammals. Nat. Med. 1:1311-1314.

36. Farooqi, I.S., et al. 2002. Beneficial effects of leptin on obesity, T cell hyporesponsiveness, and neuroendocrine/metabolic dysfunction of human congenital leptin deficiency. J. Clin. Invest. 110:1093-1103. doi:10.1172/JCI200215693.

37. Rosenbaum, M., et al. 2005. Low-dose leptin reverses skeletal muscle, autonomic, and neuroendocrine adaptations to maintenance of reduced weight. J. Clin. Invest. 115:3579-3586. doi:10.1172/ JCI25977.

38. Weissenbach, J., et al. 1980. Two interferon mRNAs in human fibroblasts: in vitro translation and Escherichia coli cloning studies. Proc. Natl. Acad. Sci. U. S. A. 77:7152-7156.

39. Hirano, T., et al. 1986. Complementary DNA for 
a novel human interleukin (BSF-2) that induces B lymphocytes to produce immunoglobulin. Nature. 324:73-76.

40. Yasukawa, K., et al. 1987. Structure and expression of human B cell stimulatory factor-2 (BSF-2/IL-6) gene. EMBO J. 6:2939-2945

41. Gadient, R.A., and Patterson, P.H. 1999. Leukemia inhibitory factor, interleukin 6 , and other cytokines using the GP130 transducing receptor: roles in inflammation and injury. Stem Cells. 17:127-137.

42. Taga, T., et al. 1989. Interleukin- 6 triggers the association of its receptor with a possible signal transducer, gp130. Cell. 58:573-581.

43. Boulay, J.L., et al. 2003. Molecular phylogeny within type I cytokines and their cognate receptors. Immunity. 19:159-163.

44. Zvonic, S., et al. 2005. Cross-talk among gp 130 cytokines in adipocytes. J. Biol. Chem. 280:33856-33863.

45. Ernst, M., and Jenkins, B.J. 2004. Acquiring signaling specificity from the receptor gp130. Trends Genet. 20:23-32.

46. Cohen, B., Novick, D., and Rubinstein, M. 1996. Modulation of insulin activities by leptin. Science. 274:1185-1188.

47. Boulton, T.G., Stahl, N., and Yancopoulos, G.D 1994. Ciliary neurotrophic factor/leukemia inhibitory factor/interleukin 6/oncostatin M family of cytokines induces tyrosine phosphorylation of a common set of proteins overlapping those induced by other cytokines and growth factors. J. Biol. Chem. 269:11648-11655.

48. Cota, D., et al. 2006. Hypothalamic mTOR signaling regulates food intake. Science. 312:927-930

49. Yokogami, K., Wakisaka, S., Avruch, J., and Reeves, S.A. 2000. Serine phosphorylation and maximal activation of STAT3 during CNTF signaling is mediated by the rapamycin target mTOR. Curr. Biol. 10:47-50.

50. Janoschek, R., et al. 2006. gp130 signaling in proopiomelanocortin neurons mediates the acute anorectic response to centrally applied ciliary neurotrophic factor. Proc. Natl. Acad. Sci. U. S. A. 103:10707-10712.

51. Schuster, B., et al. 2003. Signalling of human CNTF revisited: the interleukin-6 (IL-6) receptor can serve as an $\alpha$-receptor for ciliary neurotrophic factor (CNTF). J. Biol. Chem. 278:9528-9535.

52. Wellen, K.E., and Hotamisligil, G.S. 2005. Inflammation, stress, and diabetes. J. Clin. Invest. 115:1111-1119. doi:10.1172/JCI200525102.

53. Weisberg, S.P., et al. 2003. Obesity is associated with macrophage accumulation in adipose tissue. J. Clin. Invest. 112:1796-1808. doi:10.1172/ JCI200319246.

54. Vozarova, B., et al. 2001. Circulating interleukin-6 in relation to adiposity, insulin action, and insulin secretion. Obes. Res. 9:414-417.

55. Bastard, J.P., et al. 2000. Elevated levels of interleukin 6 are reduced in serum and subcutaneous adipose tissue of obese women after weight loss. J. Clin. Endocrinol. Metab. 85:3338-3342.

56. Lazar, M. 2005. How obesity causes diabetes: not a tall tale. Science. 307:373-375.

57. Rotter, V., Nagaev, I., and Smith, U. 2003. Interleukin-6 (IL-6) induces insulin resistance in 3T3 L1 adipocytes and is, like IL- 8 and TNFalpha, overexpressed in human fat cells from insulin-resistant subjects. J. Biol. Chem 278:45777-45785.

58. Lagathu, C., et al. 2003. Chronic interleukin-6 (IL-6) treatment increased IL- 6 secretion and induced insulin resistance in adipocyte: prevention by rosiglitazone. Biochem. Biophys. Res. Commun. 311:372-379.

59. Senn, J.J., Klover, P.J., Nowak, I.A., and Mooney, R.A. 2002. Interleukin-6 induces cellular insulin resistance in hepatocytes. Diabetes. 51:3391-3399.

60 . Senn, J.J., et al. 2003. Suppressor of cytokine signaling-3 (SOCS-3), a potential mediator of interleukin-6-dependent insulin resistance in hepatocytes.
J. Biol. Chem. 278:13740-13746.

61. Klover, P.J., Zimmers, T.A., Koniaris, L.G., and Mooney, R.A. 2003. Chronic exposure to interleukin- 6 causes hepatic insulin resistance in mice. Dia betes. 52:2784-2789.

62. Klover, P.J., Clementi, A.H., and Mooney, R.A. 2005. Interleukin- 6 depletion selectively improves hepatic insulin action in obesity. Endocrinology. 146:3417-3427.

63. Kim, H.J., et al. 2004. Differential effects of interleukin- 6 and -10 on skeletal muscle and liver insulin action in vivo. Diabetes. 53:1060-1067.

64. Kristiansen, O.P., and Mandrup-Poulsen, T. 2005 Interleukin- 6 and diabetes: the good, the bad, or the indifferent? Diabetes. 54(Suppl. 2):S114-S124.

65. Carey, A.L., and Febbraio, M.A. 2004. Interleukin-6 and insulin sensitivity: friend or foe? Diabetologia. 47:1135-1142.

66. Flores, M.B., et al. 2006. Exercise improves insulin and leptin sensitivity in hypothalamus of wistar rats. Diabetes. 55:2554-2561.

67. Kelly, M., et al. 2004. AMPK activity is diminished in tissues of IL- 6 knockout mice: the effect of exercise. Biochem. Biophys. Res. Commun. 320:449-454.

68. Al-Khalili, L., et al. 2006. Signaling specificity of interleukin- 6 action on glucose and lipid metabolism in skeletal muscle. Mol. Endocrinol. 20:3364-3375.

69. Carey, A.L., et al. 2006. IL-6 increases insulin stimulated glucose disposal in humans and glucose uptake and fatty acid oxidation in vitro via AMPK. Diabetes. 55:2688-2697.

70. Petersen, E.W., et al. 2005. Acute IL-6 treatment increases fatty acid turnover in elderly humans in vivo and in tissue culture in vitro. Am. J. Physiol. Endocrinol. Metab. 288:E155-E162.

71. Bruce, C.R., and Dyck, D.J. 2004. Cytokine regulation of skeletal muscle fatty acid metabolism: effect of interleukin- 6 and tumor necrosis factor-alpha. Am. J. Physiol. Endocrinol. Metab. 287:E616-E621.

72. van Hall, G., et al. 2003. Interleukin-6 stimulates lipolysis and fat oxidation in humans. J. Clin. Endocrinol. Metab. 88:3005-3010.

73. Weigert, C., et al. 2004. Palmitate, but not unsaturated fatty acids, induces the expression of interleukin- 6 in human myotubes through proteasomedependent activation of nuclear factor-kappaB. J. Biol. Chem. 279:23942-23952.

74. Weigert, C., et al. 2005. Interleukin-6 (IL-6) acts as insulin sensitizer on glycogen synthesis in human skeletal muscle cells by phosphorylation of Ser-473 of Akt. Am. J. Physiol. Endocrinol. Metab. 289:E251-E257.

75. Weigert, C., et al. 2006. Direct cross-talk of interleukin- 6 and insulin signal transduction via insulin receptor substrate- 1 in skeletal muscle cells. J. Biol. Chem. 281:7060-7067.

76. Adler, R., Landa, K.B., Manthorpe, M., and Varon S. 1979. Cholinergic neuronotrophic factors: intraocular distribution of trophic activity for ciliary neurons. Science. 204:1434-1436.

77. Lin, F.-L.H., et al. 1989. Purification, cloning and expression of ciliary neurotrophic factor. Science. 246:1023-1025

78. Stöckli, K.A., et al. 1989. Molecular cloning, expression, and regional distribution of rat ciliary neurotrophic factor. Nature. 342:920-923.

79. Sleeman, M.W., et al. 2000. The ciliary neurotrophic factor and its receptor, CNTFR $\alpha$. Pharm. Acto Helvet. 74:265-272.

80. Davis, S., et al. 1993. Released form of CNTF receptor alpha component as a soluble mediator of CNTF responses. Science. 259:1736-1739.

81. Chen, X., et al. 2005. Dedifferentiation of adult human myoblasts induced by ciliary neurotrophic factor in vitro. Mol. Biol. Cell. 16:3140-3151.

82. Guilett, C., et al. 1999. Ciliary neurotrophic factor is a regulator of muscular strength in aging. J. Newrosci. 19:1257-1262.
83. ALS CNTF Treatment Study Group. 1996. A double blind placebo-controlled clinical trial of subcutaneous recombinant ciliary neurotrophic factor (rH-CNTF) in amyotrophic lateral sclerosis. Neurology. 46:1244-1249.

84. Gloaguen, I., et al. 1997. Ciliary neurotrophic factor corrects obesity and diabetes associated with leptin deficiency and resistance. Proc. Natl. Acad. Sci.U.S. A. 94:6456-6461.

85. Lambert, P.D., et al. 2001. Ciliary neurotrophic factor activates leptin-like pathways and reduces body fat, without cachexia or rebound weight gain, even in leptin-resistant obesity. Proc. Natl. Acad. Sci.U. S. A. 98:4652-4657.

86. Kalra, S.P. 2001. Circumventing leptin resistance for weight control. Proc. Natl. Acad. Sci. U. S. A. 98:4279-4281.

87. Kelly,J.F., et al. 2004. Ciliary neurotrophic factor and leptin induce distinct patterns of immediate early gene expression in the brain. Diabetes. 53:911-920.

88. Prima, V., et al. 2004. Differential modulation of energy balance by leptin, ciliary neurotrophic factor, and leukemia inhibitory factor gene delivery: microarray deoxyribonucleic acid-chip analysis of gene expression. Endocrinology. 145:2035-2045.

89. Kokoeva, M.V., Yin, H., and Flier, J.S. 2005. Neurogenesis in the hypothalamus of adult mice: potential role in energy balance. Science. 310:679-683.

90. Cowley, M.A., et al. 2003. The distribution and mechanism of action of ghrelin in the CNS demonstrates a novel hypothalamic circuit regulating energy homeostasis. Neuron. 37:649-661.

91. Vogel, G. 2005. Does brain cell growth drive weight loss? Science. 310:602.

92. Andersson, U., et al. 2004. AMP-activated protein kinase plays a role in the control of food intake. J. Biol. Chem. 279:12005-12008.

93. Steinberg, G.R., et al. 2006. Ciliary neurotrophic factor suppresses hypothalamic AMP-kinase signaling in leptin-resistant obese mice. Endocrinology. 147:3906-3914

94. Ott, V., et al. 2002. Direct effects of ciliary neurotrophic factor on brown adipocytes: evidence for a role in peripheral regulation of energy homeostasis. J. Endocrinol. 173:R1-R8.

95. Zvonic, S., et al. 2003. The regulation and activation of ciliary neurotrophic factor signaling proteins in adipocytes. J. Biol. Chem. 278:2228-2235.

96. Ahima, R.S. 2006. Overcoming insulin resistance with CNTF. Nat. Med. 12:511-512.

97. Krssak, M., et al. 1999. Intramyocellular lipid concentrations are correlated with insulin sensitivity in humans: a 1H NMR spectroscopy study. Diabetologia. 42:113-116.

98. Kim, J.K., et al. 2004. PKC- $\theta$ knockout mice are protected from fat-induced insulin resistance. J. Clin. Invest. 114:823-827. doi:10.1172/JCI200422230.

99. Cai, D., et al. 2005. Local and systemic insulin resistance resulting from hepatic activation of IKK-beta and NF-kappaB. Nat. Med. 11:183-190.

100. Hirosumi, J., et al. 2003. A central role for JNK in obesity and insulin resistance. Nature. 420:333-336.

101. Watt, M.J., Hevener, A., Lancaster, G.I., and Febbraio, M.A. 2006. Ciliary neurotrophic factor prevents acute lipid-induced insulin resistance by attenuating ceramide accumulation and phosphorylation of JNK in peripheral tissues. Endocrinology. 147:2077-2085.

102. Sleeman, M.W., et al. 2003. Ciliary neurotrophic factor improves diabetic parameters and hepatic steatosis and increases basal metabolic rate in $\mathrm{db} / \mathrm{db}$ mice. Proc. Natl. Acad. Sci. U. S. A. 100:14297-14302.

103. Bluher, S., et al. 2004. Ciliary neurotrophic factorAx15 alters energy homeostasis, decreases body weight, and improves metabolic control in dietinduced obese and UCP1-DTA mice. Diabetes. 53:2787-2796.

104.Irrcher, I., Kirwan, A.F., and Hood, D.A. 2006. 
AMP-activated protein kinase-regulated activation of the PGC- $1 \alpha$ promoter in skeletal muscle cells [abstract]. FASEB J. 20:A389.

105. Petersen, K.F., et al. Impaired mitochondrial activity in the insulin-resistant offspring of patients with type 2 diabetes. N. Engl. J. Med. 350:664-671.

106. Mootha, V., et al. 2003. PGC-1alpha-responsive genes involved in oxidative phosphorylation are coordinately downregulated in human diabetes. Nat. Genet. 34:267-273.

107. Takahashi, R., et al. 1994. A null mutation in the human CNTF gene is not causally related to neu- rological diseases. Nat. Genet. 7:79-84.

108. Roth, S.M., et al. 2001. CNTF genotype is associated with muscular strength and quality in humans across the adult age span. J. Appl. Physiol. 90:1205-1210.

109.Munzberg, H., et al. 1998. Screening for variability in the ciliary neurotrophic factor (CNTF) gene: no evidence for association with human obesity. Exp. Clin. Endocrinol. Diabetes. 106:108-112.

110.O'Dell, S.D., et al. 2002. Null mutation in human ciliary neurotrophic factor gene confers higher body mass index in males. Eur. J. Hum. Genet. 10:749-752.
111.Ettinger, M.P., et al. 2003. Recombinant variant of ciliary neurotrophic factor for weight loss in obese adults: a randomized, dose-ranging study. JAMA. 289:1826-1832.

112.Kallen, K.J., et al. 1999. Receptor recognition sites of cytokines are organized as exchangeable modules. Transfer of the leukemia inhibitory factor receptor-binding site from ciliary neurotrophic factor to interleukin-6. J. Biol. Chem. 274:11859-11867.

113.Scott, D.L., and Kingsley, G.H. 2006. Tumor necrosis factor inhibits rheumatoid arthritis. N. Engl. J. Med. 355:704-712. 